\title{
Influenza Management via Direct to Consumer Telemedicine: an Observational Study
}

\author{
Michael B. Rothberg, MD, MPH and Kathryn A. Martinez, PhD, MPH(D)
}

$\mathrm{J}$ Gen Intern Med 35(10):3111-3

DOI: $10.1007 / \mathrm{s} 11606-020-05640-5$

(c) Society of General Internal Medicine 2020

\section{BACKGROUND}

Oseltamivir can shorten duration of influenza symptoms, but only if administered within $48 \mathrm{~h} .{ }^{1}$ In primary care, only $20 \%$ of patients receive oseltamivir, due to false negative tests and patients presenting too late. ${ }^{2}$ By connecting patients with physicians $24 \mathrm{~h}$ a day, direct to consumer (DTC) telemedicine could solve these problems, without exposing others to infection. Whether DTC telemedicine improves treatment rates or treatment appropriateness is unknown.

\section{OBJECTIVE}

The objective of this study was to characterize care seeking, diagnosis, and treatment for influenza in a large DTC telemedicine service.

\section{METHODS AND FINDINGS}

We describe encounters from a nationwide telemedicine platform, ${ }^{3}$ conducted between July 2016 and August 2018. Patients stated their call reason using free text and provided age, gender, and insurance information. To assess appropriateness, we described patterns of care by epidemic status and assessed patient, physician, and encounter characteristics associated with an influenza diagnosis and oseltamivir prescription. Epidemics were identified using the Center for Disease Control's Weekly U.S. Influenza Map, ${ }^{4}$ with encounters categorized as occurring during an epidemic or not. Oseltamivir prescriptions were identified via National Drug Codes. Using mixed effects logistic regression, we estimated the odds of oseltamivir prescription among patients seeking care for influenza, accounting for clustering by physician.

During the study period, 8112 patients called for "influenza." Of these, 3104 (38\%) received an influenza diagnosis (53\% during epidemics versus $11 \%$ during non-epidemics).

Received October 8, 2019

Revised November 19, 2019

Accepted January 3, 2020

Published online January 9, 2020
Of all influenza diagnoses $(n=8055), 88 \%$ were during epidemics. Following diagnosis, oseltamivir prescription did not vary by epidemic status $(80 \%$ during epidemics versus $78 \%$ during non-epidemics, $p=0.157$ ). Table 1 describes sample characteristics and associations with diagnosis and treatment.

Thirty-five percent of patients seeking care for influenza received it during weekends or after hours. Median wait time was $4.6 \mathrm{~min}$ (interquartile range (IQR) 1.910.3 ) and median visit length was 4.9 min (IQR 3.57.4). Had they not used telemedicine, $87 \%$ said they would have gone to a doctor's office, urgent care, or the emergency room.

Among patients seeking care for influenza, calling during an epidemic was strongly associated with oseltamivir prescription (aOR 7.78; 95\% CI 6.86-9.27) (Table 2), while not reporting insurance was negatively associated (aOR 0.68 ; 95\% CI 0.57-0.82).

\section{DISCUSSION}

In this observational study of DTC telemedicine, patients frequently sought care for influenza and most diagnoses were made during epidemics. Early diagnosis is important both for treatment and to avoid spread of infection. During an epidemic, diagnosis can be made based on symptoms alone. ${ }^{5,6}$ Rapid testing is not required and has a false negative rate of $>40 \% .^{2}$ Treatment is most effective if provided within $12 \mathrm{~h}$ of symptom onset, ${ }^{1}$ yet $36 \%$ of primary care patients present after $48 \mathrm{~h}$ when treatment is no longer effective. ${ }^{2}$ This problem may be overcome by the rapid access afforded by telemedicine. Indeed, onethird of our patients sought care after hours or on weekends, when doctor's offices aren't typically open, and completed their visits in $<20 \mathrm{~min}$. Without telemedicine, most patients would have sought care in public venues, likely delaying care and potentially infecting others. Approximately $40 \%$ of patients calling with "influenza" were prescribed oseltamivir, approximately double that observed in primary care.

During non-epidemics, when the pretest probability of influenza is low, diagnosis requires rapid testing, which is not available via telemedicine. Because oseltamivir is expensive and $10 \%$ of patients experience nausea, treatment should be 
Table 1 Sample Characteristics of Patients Seeking Care for Influenza, Association with Diagnosis and Oseltamivir Prescription

\begin{tabular}{|c|c|c|c|c|c|c|}
\hline & \multicolumn{3}{|c|}{ Call reason was influenza $(N=8112)$} & \multicolumn{3}{|c|}{ Diagnosed with influenza $(N=8055)$} \\
\hline & Overall $N(\%)$ & $N(\%)$ diagnosed & & Overall $N(\%)$ & $\begin{array}{l}\text { Prescribed } \\
\text { oseltamivir } N(\%)\end{array}$ & $p$ value* \\
\hline \multicolumn{7}{|l|}{ Influenza season } \\
\hline Yes & $5344(66)$ & $2806(53)$ & \multirow{2}{*}{$<0.001$} & $7113(88)$ & $5683(80)$ & \multirow[t]{2}{*}{0.157} \\
\hline No & $2768(34)$ & $298(11)$ & & $942(12)$ & $734(78)$ & \\
\hline \multicolumn{7}{|l|}{ Weekend or after hours } \\
\hline No & $5274(65)$ & $2009(38)$ & \multirow{2}{*}{0.664} & $5195(64)$ & $4113(79)$ & \multirow[t]{2}{*}{0.139} \\
\hline Yes & $2838(35)$ & $1095(39)$ & & $2860(36)$ & $2304(81)$ & \\
\hline \multicolumn{7}{|l|}{ Patient gender } \\
\hline Female & $4724(58)$ & $1768(37)$ & \multirow[t]{2}{*}{0.067} & $4570(57)$ & $3635(80)$ & \multirow[t]{2}{*}{0.750} \\
\hline Male & $3388(42)$ & $1336(39)$ & & $3485(43)$ & $2782(80)$ & \\
\hline \multicolumn{7}{|l|}{ Patient age } \\
\hline$<19$ years & $967(12)$ & $457(47)$ & \multirow[t]{4}{*}{$<0.001$} & $1319(16)$ & $1039(79)$ & \multirow[t]{4}{*}{0.297} \\
\hline 19-39 years & $3816(47)$ & $1395(37)$ & & $3613(45)$ & $2867(79)$ & \\
\hline $40-59$ & $2959(36)$ & $1109(37)$ & & $2802(35)$ & $2262(81)$ & \\
\hline 60 and older & $370(5)$ & $143(39)$ & & $321(4)$ & $249(78)$ & \\
\hline \multicolumn{7}{|c|}{ Reported insurance information } \\
\hline Yes & $7124(88)$ & $2814(39)$ & \multirow[t]{2}{*}{$<0.001$} & $7371(92)$ & $5881(80)$ & \multirow[t]{2}{*}{0.376} \\
\hline No & $988(12)$ & $290(29)$ & & $684(8)$ & $536(78)$ & \\
\hline \multicolumn{7}{|l|}{ Patient region $^{\dagger}$} \\
\hline Northeast & $1183(15)$ & $383(22)$ & \multirow[t]{4}{*}{$<0.001$} & $1023(13)$ & $779(76)$ & \multirow[t]{4}{*}{$<0.001$} \\
\hline Midwest & $2017(25)$ & $755(37)$ & & $2166(27)$ & $1706(79)$ & \\
\hline South & $3354(41)$ & $1501(45)$ & & $3652(45)$ & $2984(82)$ & \\
\hline West & $1558(19)$ & $465(30)$ & & $1214(15)$ & $948(78)$ & \\
\hline \multicolumn{7}{|l|}{ Physician specialty } \\
\hline Family Medicine & $5341(66)$ & $1978(37)$ & \multirow[t]{4}{*}{$<0.001$} & $5170(64)$ & $4224(82)$ & \multirow[t]{4}{*}{$<0.001$} \\
\hline Internal Medicine & $1858(23)$ & $768(41)$ & & $1932(24)$ & $1474(76)$ & \\
\hline Emergency Medicine & $615(8)$ & $220(36)$ & & $637(8)$ & $483(76)$ & \\
\hline Pediatrics & $298(4)$ & $138(46)$ & & $316(4)$ & $236(75)$ & \\
\hline \multicolumn{7}{|l|}{ Physician region $^{\dagger}$} \\
\hline Northeast & $1625(20)$ & $501(16)$ & \multirow[t]{4}{*}{$<0.001$} & $1323(16)$ & $1041(79)$ & \multirow[t]{4}{*}{0.163} \\
\hline Midwest & $1797(22)$ & $790(25)$ & & $2245(28)$ & $1761(78)$ & \\
\hline South & $3252(40)$ & $1361(44)$ & & $3388(42)$ & $2731(81)$ & \\
\hline West & $1438(18)$ & $452(15)$ & & 1099 (14) & $884(80)$ & \\
\hline
\end{tabular}

*p value from chi-square test

tBased on U.S. Census categories

Table 2 Adjusted Odds of Being Prescribed Oseltamivir Among Patients Stating Their Call Reason Was Influenza

\begin{tabular}{|c|c|c|}
\hline$(N=8112)$ & aOR & $95 \% \mathrm{CI}$ \\
\hline \multicolumn{3}{|l|}{ Influenza season } \\
\hline No & 1.00 & \\
\hline Yes & 7.98 & $6.86-9.27$ \\
\hline \multicolumn{3}{|l|}{ Patient age (years) } \\
\hline 18 and under & 1.39 & $1.16-1.68$ \\
\hline $19-39$ & 1.00 & \\
\hline $40-59$ & 0.91 & $0.80-1.02$ \\
\hline 60 and over & 0.86 & $0.67-1.12$ \\
\hline \multicolumn{3}{|l|}{ Patient gender } \\
\hline Female & 1.00 & \\
\hline Male & 1.10 & $0.98-1.23$ \\
\hline \multicolumn{3}{|c|}{ Reported insurance information } \\
\hline Yes & 1.00 & \\
\hline No & 0.68 & $0.57-0.82$ \\
\hline \multicolumn{3}{|l|}{ Patient region* } \\
\hline Northeast & 1.00 & \\
\hline Midwest & 0.96 & $0.78-1.19$ \\
\hline South & 1.42 & $1.17-1.73$ \\
\hline West & 0.80 & $0.63-1.00$ \\
\hline \multicolumn{3}{|l|}{ Physician region* } \\
\hline Northeast & 1.00 & \\
\hline Midwest & 1.23 & $0.60-1.12$ \\
\hline South & 1.09 & $0.49-1.21$ \\
\hline West & 1.10 & $0.72-1.66$ \\
\hline \multicolumn{3}{|l|}{ Physician specialty } \\
\hline Family Medicine & 1.00 & \\
\hline Internal Medicine & 0.82 & $0.60-1.12$ \\
\hline Emergency Medicine & 0.77 & $0.49-1.21$ \\
\hline Pediatrics & 0.35 & $0.19-0.65$ \\
\hline
\end{tabular}

*Based on U.S. Census categories reserved for patients with a high probability of infection. Most treatment in our study appeared guideline-concordant, but $12 \%$ of oseltamivir was prescribed out of season, exposing patients to expense and side effects with little chance of benefit. However, that patients who did not report insurance information were less likely to get it shows that telemedicine physicians are sensitive to patient costs.

Our study is limited by the data available. We did not have access to physician notes and so do not know specific symptoms, duration of illness, or co-morbidities. We also do not know whether patients filled the prescriptions.

In treating influenza, time is of the essence. During yearly epidemics, telemedicine allows for rapid, appropriate treatment, while limiting public exposure to infected individuals. Efforts should be made to increase use of telemedicine for this purpose.

Corresponding Author: Kathryn A. Martinez, PhD, MPH; Cleveland Clinic Center for Value-Based Care Research, 9500 Euclid Ave, G10, Cleveland, OH 99150, USA (e-mail: martink12@ccf.org).

\section{Compliance with Ethical Standards:}

Conflict of Interest: The authors have no conflicts of interest to disclose. 


\section{REFERENCES}

1. Aoki F, Macleod M, Paggiano $\mathbf{P}$, et al. Early administration of oral oseltamivir increases the benefits of influenza treatment. $J$ Antimicrob Chemother. 2003;50(1): 123-129.

2. Fowlkes AL, Steffens A, Reed C, Temte JL, Campbell AP, Influenza Incidence Surveillance Project Working Group. Influenza Antiviral Prescribing Practices and the Influence of Rapid Testing Among Primary Care Providers in the US, 2009-2016. Open forum Infect Dis. 2019;6(6):ofz192. doi:https://doi.org/10.1093/ofid/ofz192

3. Martinez KA, Rood M, Jhangiani N, Rose S, Boissy A, Rothberg MB Patterns of Use and Correlates of Patient Satisfaction with a Large Nationwide Direct to Consumer Telemedicine Service. J Gen Intern Med.
4. Centers for Disease Control and Prevention. Weekly US Map: Influenza Summary Update. https://www.cdc.gov/flu/weekly/usmap.htm. Accessed January 4, 2019.

5. Call SA, Vollenweider MA, Hornung CA, Simel DL, McKinney WP. Does This Patient Have Influenza? JAMA. 2005;293(8):987. doi:https://doi.org/ 10.1001/jama.293.8.987

6. Monto A, Gravenstein S, Elliott M, Colopy M, Schweinle J. Clinical signs and symptoms predicting influenza infection. Arch Intern Med. 2000; Nov 27; 16(21):3243-3247.

Publisher's Note Springer Nature remains neutral with regard to jurisdictional claims in published maps and institutional affiliations. 